\title{
On Multiple Access Random Medium Access Control
}

\author{
Tao Cui and Tracey Ho \\ Department of Electrical Engineering \\ California Institute of Technology \\ Pasadena, CA, USA 91125 \\ Email: \{taocui, tho\}@caltech.edu
}

\begin{abstract}
In this paper, we develop a new class of medium access control protocol, which allows each user to transmit at different data rates chosen randomly from an appropriately determined set of rates. By using successive interference cancellation, multiple packets can be received simultaneously. In slotted Aloha type Gaussian networks, we show that the achievable total throughput of the proposed protocol is at least a constant fraction of the mac sum rate when the number of transmission rates at each node is equal to the number of users in the network. We also study the case when only a limited number of transmission rates is available at each node. Extension to rate splitting is discussed. Simulation results show that the proposed protocol can achieve a significant throughput gain over the conventional Aloha.
\end{abstract}

\section{INTRODUCTION}

The medium access control (MAC) layer decides when competing nodes may access the shared medium. Different from schedule-based medium access requiring a central authority, contention based MAC by using e.g., random access, is a distributed strategy to access and share the medium, which is popular in wireless networks. The MAC layer is traditionally designed separately from the physical layer. Most conventional random access protocols such as Aloha [1] and carrier sense multiple access (CSMA) [2] assume simple collision models, where the channel is noiseless, and reception failure is caused by collisions among users. Though the analysis and protocol design are simple in the collision model, the maximum achievable throughput of this model is limited. With more sophisticated physical layer approaches, simultaneous reception of multiple packets is possible, for example, by using code division multiple access (CDMA) and multiuser detection. In order to represent such random access systems, a model for a channel with multipacket reception capability (MPR) with its stability property has been developed in [3]. A decentralized MAC protocol is proposed in [4]. In these work [3], [4], it is shown that the achievable throughput by using MPR is higher than that by using Aloha.

In MPR, each node transmits only at a single rate. On the other hand, in a multiple access system with $N$ users and one base station, this system can be considered to be a multiple access channel (mac) [5]. If each user transmits with power $P$ and the noise power at the base station is $\sigma^{2}$, the maximum information theoretic sum rate of all users is $\frac{1}{2} \log \left(1+\frac{N P}{\sigma^{2}}\right)$, which can be achieved with multirate transmission capability and successive interference cancelation (SIC).

This work has been supported in part by DARPA grant N66001-06-C2020, Caltech's Lee Center for Advanced Networking, the Okawa Foundation Research Grant and a gift from Microsoft Research.
In this paper, we develop a new class of MAC protocol by applying a SIC based approach at the MAC layer. The MPR model in [3], [4] is generalized by allowing each user to transmit at different data rates chosen randomly from an appropriately determined set of rates. In each time slot, each user transmits a packet at a randomly chosen data rate. By using SIC [5], multiple packets can be received simultaneously.

In slotted Aloha type networks with Gaussian channels, we show that the achievable sum rate of the new protocol using decentralized control is at least a constant fraction of that achievable by using centralized control, i.e., $\frac{C}{2} \log \left(1+\frac{N P}{\sigma^{2}}\right)$, $0<C<1$, where $C$ can be interpreted as the distributive loss due to contention and lack of cooperation between users. This result suggests that the total throughput increases with $N$ as opposed to Aloha where the total throughput decreases with $N$. Finally, we consider extension to rate splitting [6]. Rate splitting has been applied to Aloha in [7], [8]. We propose a new class of rate splitting algorithm which generalizes that in [8]. It is also shown to improve the achievable throughput. Our simulation results support our analysis and show that the proposed protocol achieves a significant throughput gain over conventional Aloha in Aloha type networks.

\section{A Motivating Example}

We first consider a simple example to motivate this new MAC model. There are $N=2$ users in the network, where user $i$ 's transmitted signal is $X_{i}, i=1,2$. Both users are saturated, i.e., they always have packets to send. The received signal at the base station is

$$
Y=X_{1}+X_{2}+W
$$

where the average power of $X_{i}, i=1,2$ is $P$ and the additive white Gaussian noise (AWGN) $W$ is of zero mean and variance $\sigma^{2}$. Let $S_{i}$ be the data sending rate of user $i$. From [5], the capacity region of the multiple access channel (1) is

$$
\begin{aligned}
S_{1}<I\left(X_{1} ; Y \mid X_{2}\right) & =\frac{1}{2} \log \left(1+\frac{P}{\sigma^{2}}\right), \\
S_{2}<I\left(X_{2} ; Y \mid X_{1}\right) & =\frac{1}{2} \log \left(1+\frac{P}{\sigma^{2}}\right), \\
S_{1}+S_{2}<I\left(X_{1}, X_{2} ; Y\right) & =\frac{1}{2} \log \left(1+\frac{2 P}{\sigma^{2}}\right),
\end{aligned}
$$

where $I(X ; Y)$ is the mutual information between random variables $X$ and $Y$ [5]. With a centralized controller, the maximum achievable sum rate is $R_{\mathrm{c}-\mathrm{mac}}=\frac{1}{2} \log \left(1+\frac{2 P}{\sigma^{2}}\right)$. The corner point can be achieved by decoding user 2 's signal 
first, treating user 1's signal as noise. The base station can then subtract the decoded signal from $Y$ and decode user 1's signal. This is called successive interference cancelation. Similarly, corner point $\mathrm{C}$ can be achieved. The points on the line connecting $\mathrm{B}$ and $\mathrm{C}$ can be achieved by time sharing between $\mathrm{B}$ and $\mathrm{C}$ or by using rate splitting [6].

Using Aloha, we assume that each user transmits at rate $\frac{1}{2} \log \left(1+\frac{P}{\sigma^{2}}\right)$ with probability $p$ and remains idle with probability $1-p$. The achievable sum rate of Aloha is

$$
R_{\text {Aloha }}(p)=p(1-p) \log \left(1+\frac{P}{\sigma^{2}}\right),
$$

whose maximum is attained at $p=\frac{1}{2}$. The maximum achievable throughput is

$$
R_{\mathrm{Aloha}}^{*}=\frac{1}{4} \log \left(1+\frac{P}{\sigma^{2}}\right) .
$$

To achieve the maximum sum rate $R_{\mathrm{c}-\mathrm{mac}}$ without using time sharing or rate splitting, the two users should operate cooperatively at corner point B or C, i.e., one of the user should transmit at rate $R_{1}=\frac{1}{2} \log \left(1+\frac{P}{\sigma^{2}}\right)$ and the other one transmits at rate $R_{2}=\frac{1}{2} \log \left(1+\frac{P}{P+\sigma^{2}}\right)$. Without coordination, each user transmits at rate $R_{1}$ with probability $p$ and at rate $R_{2}$ with probability $1-p$. When both users transmit at rate $R_{1}$, the rate pair is outside the capacity region (2). Thus, the receiver cannot decode both packets. In all other cases, by using SIC, both users' packets can be decoded. The average achievable rate by using the decentralized mac is

$$
R_{\mathrm{d}-\operatorname{mac}}(p)=2\left(R_{1}+R_{2}\right) p(1-p)+2 R_{2}(1-p)^{2} .
$$

$R_{\text {mac-SIC }}$ is maximized when $p=\frac{R_{1}-R_{2}}{2 R_{1}}$ and the maximum throughput is

$$
R_{\mathrm{d}-\mathrm{mac}}^{*}=\frac{\left(R_{1}+R_{2}\right)^{2}}{2 R_{1}}>\frac{1}{2}\left(R_{1}+R_{2}\right) .
$$

We thus have

$$
R_{\mathrm{c}-\mathrm{mac}}>R_{\mathrm{d}-\mathrm{mac}}^{*}>\frac{1}{2} R_{\mathrm{c}-\mathrm{mac}}, \quad R_{\mathrm{d}-\mathrm{mac}}^{*}>R_{\mathrm{Aloha}}^{*} .
$$

Therefore, the proposed new MAC protocol can achieve at least $50 \%$ of the throughput using a centralized controller and its throughput is always greater than Aloha. It can be shown that the proposed strategy is actually optimal over all possible transmission strategies when rate splitting is not used and both users adopt the same transmission strategy for fairness.

Note that using the MPR model both users randomly attempt to transmit at a single fixed rate. When both users transmit at $R_{1}$, the MPR model reduces to Aloha. When the rate is $R_{2}$, the two users can transmit simultaneously. However, the achievable throughput is less than Aloha when $P \gg \sigma^{2}$. Therefore, by enabling multirate data transmission at each node and using SIC, the proposed protocol outperforms both Aloha and MPR and has comparable performance with a centralized controller.

\section{Multiple Access MAC in Aloha Type Networks}

\section{A. MAC on $A W G N$ mac}

Let $X_{i}$ be the transmitted signal by user $i$ and $Y$ be the signal received by the receiver. We use the model

$$
Y=\sum_{i=1}^{N} h_{i} X_{i}+W,
$$

where the power of user $i$ is $P_{i}$ and the AWGN $W$ is of zero mean and variance $\sigma^{2}$. We first consider a homogenous system with $P_{i}=P$ and $h_{i}=1, \forall i \in\{1, \ldots, N\}$. Let $S_{i}$ be the data rate of user $i$. From [5], the capacity region of mac with a centralized controller is

$$
\sum_{i \in \mathcal{U}} S_{i} \leq I\left(X(\mathcal{U}) ; Y \mid X\left(\mathcal{U}^{c}\right)\right), \forall \mathcal{U} \subseteq\{1,2, \ldots, N\},
$$

for some product distribution $p_{1}\left(x_{1}\right) p_{2}\left(x_{2}\right) \cdots p_{N}\left(x_{N}\right)$, where $X(\mathcal{U})=\left\{X_{i}: i \in \mathcal{U}\right\}$ and $\mathcal{U}^{c}$ denotes the complement of $\mathcal{U}$ in $\{1,2, \ldots, N\}$. The capacity region (9) constitutes a polytope, which contains $N$ ! corner points. Each corner point corresponds to a permutation $\pi$ of the $N$ users. The receiver decodes using SIC. User $\pi(i)$ is decoded by treating users $\pi(1), \ldots, \pi(i-1)$ as noise. After decoding, the contribution of user $\pi(i)$ in $Y$ is removed. The process continues until user $\pi(1)$ 's packet is decoded. The maximum achievable sum rate with a central controller is

$$
R_{\mathrm{c}-\operatorname{mac}}(N)=\frac{1}{2} \log \left(1+\frac{N P}{\sigma^{2}}\right) .
$$

As in Section II, users want to reach a corner point distributedly to attain the maximum sum rate. In our multiple access MAC, we assume that each user is capable of transmitting at one of $N$ rates, where the $k$-th rate is

$$
R_{k}=\frac{1}{2} \log \left(1+\frac{P}{(k-1) P+\sigma^{2}}\right), k=1, \ldots, N .
$$

Note that $\sum_{k=1}^{N} R_{k}=R_{\mathrm{c}-\operatorname{mac}}(N)$. When each user chooses a different rate from $\left\{R_{k}: k=1, \ldots, N\right\}$, a corner point is attained. Let $n_{k}$ be the number of users that transmit at rate $R_{k}$. Using SIC, the packets of rate $R_{k}$ can be decoded if and only if the packets of rate less than $R_{k}$ are decoded correctly so that their contribution can be cancelled from $Y$, and the number of users transmitting at rate greater than or equal to $R_{k}$ is at most $k$, i.e., $\sum_{l=1}^{j} n_{l} \leq j, j=k, \ldots, N$, because from (11) the user at $R_{k}$ can tolerate interference level $(k-1) P$. It can be shown that using the set of rates (11) is optimal over all possible sets of rates when rate splitting is not used and all users adopt the same transmission strategy.

We can use pseudo random variables with random seeds to choose the transmission rate at each node, so that with the random seed each receiver knows each user's transmission rate at each time slot. This reduces the decoding complexity to $O(N)$ since each receiver knows the order in which to decode the users. In the absence of random seeds or other side information the receiver can first attempt to decode the lowest rate packets for each source, which has complexity $O(N)$. After cancelling the contribution of decoded signal from the received signal, the receiver tries to decode the second lowest rate packets for each source. The process repeats until the highest rate is reached. The total complexity is proportional to $O\left(N^{2}\right)$. The receiver does not need to know different transmitters' sending rates.

\section{B. Achievable Results}

In this subsection, we study the average achievable throughput of our model using the set of transmission rates (11) and compare it with $R_{\mathrm{c}-\operatorname{mac}}(N)$ in (10). Let $\mathcal{S}_{-i}=$ $\left(S_{1}, \ldots, S_{i-1}, S_{i+1}, \ldots, S_{N}\right)$ be the state of all the nodes other than node $i$, where $S_{i}$ is the transmission rate of node $i$. The average throughput of the network attained by the distributed mac strategy is 


$$
\begin{aligned}
& R_{\mathrm{d}-\mathrm{mac}}(N)=E\left\{\sum_{i=1}^{N} b_{i} S_{i}\right\} \stackrel{(a)}{=} N E\left\{b_{i} S_{i}\right\}=N E_{\mathcal{S}_{-i}}\left\{E\left\{b_{i} S_{i} \mid \mathcal{S}_{-i}\right\}\right\} \\
= & N \sum_{k=1}^{N} p_{k} R_{k} \sum_{\mathcal{S}_{-i}} \operatorname{Pr}\left(b_{i}=1 \mid\left(S_{i}=R_{k}, \mathcal{S}_{-i}\right) \operatorname{Pr}\left(\mathcal{S}_{-i}\right)\right. \\
= & N \sum_{k=1}^{N} p_{k} R_{k} \underbrace{}_{n_{n_{1}^{\prime}, \ldots, n_{N}^{\prime}} \operatorname{Pr}\left(\sum_{l=1}^{j} n_{l}^{\prime} \leq j-1, j=k, \ldots, N\right)}
\end{aligned}
$$

where $(a)$ is due to symmetry, $b_{i} \in\{0,1\}$ is an indicator whether user $i$ 's packet is decoded correctly at the receiver, $n_{k}^{\prime}=n_{k}-1$, and $n_{l}^{\prime}=n_{l}$ for $l \neq k$ denote the number of users transmitting at rate $R_{l}$ other than user $i$. Note that (12) only requires that packets with rate less than $R_{k}$ are decoded, where packets with rate higher than $R_{k}$ may be decoded incorrectly. Let $m_{k}=\sum_{l=1}^{k} n_{l}^{\prime}$. We can write $q_{k}$ as

$$
q_{k}=\sum_{\substack{m_{k}, n_{k+1}^{\prime}, \ldots, n_{N}^{\prime} \\
m_{k}+\sum_{l=k+1}^{j} n_{l}^{\prime} \leq j-1, j=k, \ldots, N}}\left(\begin{array}{c}
N-1 \\
m_{k}, n_{k+1}^{\prime}, \ldots, n_{N}^{\prime}
\end{array}\right) \prod_{l=k+1}^{N} p_{l}^{n_{l}^{\prime}}\left(\sum_{l=1}^{k} p_{l}\right)^{m_{k}} .
$$

Given $p_{k}$, it is complex to compute $q_{k}$ through (13). To circumvent this problem, we find a recursive relationship between $q_{k}$ and $q_{k-1}$. Let $A_{k}$ denote the event that the rates of all users excluding user $i$ are such that if $i$ were to send at rate $R_{k}$, its packets would be decoded correctly. Then let $q_{k}=\operatorname{Pr}\left(A_{k}\right)$. By the total probability theorem, we obtain

$$
\operatorname{Pr}\left(A_{k}\right)=\operatorname{Pr}\left(A_{k} \mid A_{k-1}\right) \operatorname{Pr}\left(A_{k-1}\right)+\operatorname{Pr}\left(A_{k} \mid A_{k-1}^{c}\right) \operatorname{Pr}\left(A_{k-1}^{c}\right) \text {. }
$$

Given $\mathcal{S}_{-i}$, if $S_{i}=R_{k-1}$ and user $i$ 's packet can be decoded at the receiver, when $S_{i}=R_{k}$ user $i$ 's packet can still be decoded because $R_{k}<R_{k-1}$, which gives $\operatorname{Pr}\left(A_{k} \mid A_{k-1}\right)=1$. On the other hand, $A_{k} \cap A_{k-1}^{c}$ means that if user $i$ were to send at rate $R_{k}$ its packets would be decoded correctly but its packets cannot be decoded if it were to send at rate $R_{k-1}$. This event occurs if and only if among the remaining $N-1$ users, $k-1$ of them transmit at rate above $R_{k}$ so that transmitting at $R_{k}$ is admissible but not at $R_{k-1}, N-k$ of them transmit at rate below $R_{k}$, and the $N-k$ users' packets can be decoded correctly. Let $\eta_{j, k}$ denote the probability that $j$ given users each transmits at rate less than or equal to $R_{k}$ and their packets can be decoded correctly at the receiver. From the definition of $R_{k}$ in (11), if a user transmits at rate $R_{N}$, the receiver can decode its packet regardless of other users' transmissions, which means $q_{N}=1$. We can thus establish the recursive relation

$$
q_{k-1}=q_{k}-\left(\begin{array}{c}
N-1 \\
k-1
\end{array}\right)\left(\sum_{j=1}^{k-1} p_{j}\right)^{k-1} \eta_{N-k, k+1}, q_{N}=1 .
$$

To compute $\eta_{j, k}$, note that there are $\left(\begin{array}{l}j \\ l\end{array}\right)$ ways to choose $l$ users out of $j$ users and let them transmit at rate $R_{k}$. The remaining $j-l$ users transmit at rates less than $R_{k}$. Assuming that $N-j$ other users transmit at rate greater than $R_{k}$, all the $j$ users' packets can be decoded if and only if $N-j+l \leq k$ and the remaining $j-l$ users' packets can be decoded. We thus have the recursive equation

$$
\eta_{j, k}=\sum_{l=0}^{k-N+j}\left(\begin{array}{l}
j \\
l
\end{array}\right) p_{k}^{l} \eta_{j-l, k+1}, \text { and } \eta_{j, N}=p_{N}^{j} .
$$

Given $p_{k}$, by using these two recurrences (15) and (16), we can evaluate the throughput efficiently by first creating a table for $\eta_{j, k}$ using (16) and then using (15) to compute $q_{k}$, which enables fast computation of achievable rate.

To find the maximum achievable asymptotic rate of the proposed scheme, we need to find the optimal $p_{k}$ by maximizing $R_{\mathrm{d}-\text { mac }}(N)$ in (12) for each $N$, which is hard to obtain in closed form. Instead, we find a lower bound on $R_{\mathrm{d}-\operatorname{mac}}(N)$ by choosing a suboptimal $p_{k}$. We have the following theorem.

Theorem 1: The maximum achievable sum rate by using distributed mac and the set of rates in (11) is $R_{\mathrm{d}-\operatorname{mac}}(N)=$ $\Theta\left(\log \left(1+\frac{N P}{\sigma^{2}}\right)\right)$. Furthermore, there exists a constant $C>0$ such that $R_{\mathrm{d}-\operatorname{mac}}(N) \geq C R_{\mathrm{c}-\operatorname{mac}}(N)$.

Proof: We consider $p_{k}=\frac{\alpha}{N-1}, k=1, \ldots, N-1$ and $p_{N}=1-\alpha$, where $0<\alpha<1$ is a constant to be determined later. Note that $q_{N}=1$ and

$$
\left(\begin{array}{c}
N-1 \\
k-1
\end{array}\right)\left(\sum_{j=1}^{k-1} p_{j}\right)^{k-1} \eta_{N-k, k+1} \leq \frac{(k-1)^{k-1}}{(k-1) !} \alpha^{k-1} .
$$

where we have used the fact that $\eta_{j, k} \leq 1$ and $\frac{(N-1) !}{(N-k) !}<(N-$ $1)^{k-1}$. Applying induction on (15), we can obtain

$$
q_{1} \geq 1-\sum_{k=2}^{N} \frac{(k-1)^{k-1}}{(k-1) !} \alpha^{k-1}>1-\sum_{k=1}^{+\infty} \frac{k^{k}}{k !} \alpha^{k} .
$$

To show the convergence of the series $\sum_{k=1}^{+\infty} \frac{k^{k}}{k !} \alpha^{k}$, we use ratio test, which needs to compute

$$
L=\lim _{k \rightarrow \infty} \frac{(k+1)^{k+1}}{(k+1) !} \alpha^{k+1} \frac{k !}{k^{k}} \alpha^{-k}=\alpha e .
$$

Therefore, if $L<1$ or $\alpha<e^{-1}, \sum_{k=1}^{+\infty} \frac{k^{k}}{k !} \alpha^{k}$ converges to a number $B(\alpha)$. It is easy to see that $B(0)=0$ and $B(\alpha)$ is a continuous and increasing function in $\alpha$. Therefore, there exists a threshold $\gamma$ such that $B(\alpha)<1$ when $0<\alpha<\gamma$. Note that $q_{1} \leq q_{2} \leq \cdots \leq q_{N}$ and $p_{N}>p_{k}, k=1, \ldots, N-1$ when $N$ is large. Thus, we have

$$
\begin{aligned}
R_{\mathrm{d}-\operatorname{mac}}(N) & =N \sum_{k=1}^{N} p_{k} q_{k} R_{k} \geq \alpha(1-B(\alpha)) \sum_{k=1}^{N} R_{k} \\
& =\frac{\alpha}{2}(1-B(\alpha)) \log \left(1+\frac{N P}{\sigma^{2}}\right) .
\end{aligned}
$$

We can find the optimal $\alpha$ by maximizing $\alpha(1-B(\alpha))$, which is the solution of

$$
\sum_{k=1}^{+\infty} \frac{k^{k}}{k !}(k+1) \alpha^{k}=1
$$

By solving (21) numerically, we find that $\alpha=0.2011$ and

$$
R_{\mathrm{d}-\operatorname{mac}}(N) \geq \frac{0.13}{2} \log \left(1+\frac{N P}{\sigma^{2}}\right)=0.13 R_{\mathrm{c}-\operatorname{mac}}(N) .
$$

On the other hand, $R_{\mathrm{d}-\operatorname{mac}}(N)$ is less than $R_{\mathrm{c}-\operatorname{mac}}(N)$, the achievable rate of a centralized controller. Therefore, we show that $R_{\mathrm{d}-\operatorname{mac}}(N)=\Theta\left(\log \left(1+\frac{N P}{\sigma^{2}}\right)\right)$.

The constant $C$ in Theorem 1 can be interpreted as the distributive loss due to contention and lack of cooperation between users. By choosing $p_{1}=\frac{1}{N}, p_{2}=, \cdots,=p_{N-1}=0$, and $p_{N}=\frac{N-1}{N}$, it is easy to see that the throughput of the proposed model is greater than that of Aloha. We thus obtain the following corollary.

Corollary 1: Let $R_{\text {Aloha }}(N)$ be the throughput of Aloha. We have $R_{\mathrm{d}-\mathrm{mac}}(N)>R_{\text {Aloha }}(N)$. 
Theorem 1 suggests that the total throughput of the new MAC model increases with increasing $N$ as opposed to Aloha where the total throughput decreases in $N$. Actually, Aloha can be considered to be a distributed implementation of TDMA while our approach is a distributed implementation of mac.

\section{Fixed Number of Transmission Rates}

The $N$-rate model can achieve a fraction of the achievable rate by a centralized controller. On one hand, in practice, MAC layer is built into firmware and the set of rates cannot be altered as the number of users in the network varies. On the other hand, the first few $R_{k}$ 's in (11) are typically significantly larger than the other rates in practical scenarios. Motivated by these two factors, we thus generalize the $N$-rate model to the case with $K$ rates, where $K$ is a fixed number that does not vary with $N$. Each node is capable of transmitting at one of $K$ rates, $R_{1}, \ldots, R_{K}$ and $R_{1}>R_{2}>\cdots>R_{K}$. Assuming that $n_{k}$ nodes transmit at rate $R_{k}$, the packets of rate $R_{k}$ can be decoded if and only if $\sum_{l=1}^{j} n_{l} \leq \omega_{j}, j=k, \ldots, K$, where $\omega_{k}$ is the maximum number of users with transmission rates higher than $R_{k}$ such that users with transmission rate $R_{k}$ can decode its packets. The decoding complexity is proportional to $N K$.

In this subsection, we consider $R_{k}=\frac{1}{2} \log \left(1+\frac{P}{(k-1) P+\sigma^{2}}\right)$, $k=1, \ldots, K-1$ and $R_{K}=\frac{1}{2} \log \left(1+\frac{P}{(N-1) P+\sigma^{2}}\right)$ for simplicity, which gives $\omega_{k}=k, k=1, \ldots, K-1$ and $\omega_{K}=N$. Even though the optimal $p_{k}$ maximizing the average throughput may depend on $R_{k}$ and $N$ in a complicated way which does not lead to simple practical protocol design, the following theorem shows that the optimal $p_{k}$ has a simple form asymptotically as $N \rightarrow$ $+\infty$.

Theorem 2: When $R_{k}=\frac{1}{2} \log \left(1+\frac{P}{(k-1) P+\sigma^{2}}\right), k=1, \ldots, K-$ 1 and $R_{K}=\frac{1}{2} \log \left(1+\frac{P}{(N-1) P+\sigma^{2}}\right)$, and $\omega_{k}=k, k=1, \ldots, K-1$ and $\omega_{K}=N$, the optimal $p_{k}$ maximizing the average throughput satisfies $\lim _{N \rightarrow+\infty} N p_{k}=\xi_{k}, k=1, \ldots, K-1$, where $\xi_{k}$ are constants depending only on $R_{1}, \ldots, R_{K}$. In other words, $p_{k}=\frac{\xi_{k}}{N}$ maximizes the average throughput asymptotically.

Theorem 2 removes the dependence of $N$ in optimizing $p_{k}$, which facilities distributed dynamic algorithm design, e.g., optimization on $\xi_{k}$ is done only once and the resulting $\xi_{k}$ can be applied for any $N$. By using game theoretic framework in [9], we do not even need to know $N$ given $\xi_{k}$. The multiple access MAC in Aloha type networks can be extended to wireless LAN.

When $K$ is small, we can obtain $\xi_{k}$ in closed form. We give an example in the following.

Example $(K=2)$ : When $K=2$, the average throughput is

$$
N R_{1} p(1-p)^{N-1}+N R_{2}(1-p),
$$

where $p$ is the probability of choosing $R_{1}$ and $\omega_{1}=1, \omega_{2}=N$. Maximizing (23), we obtain the optimal $p$, whose closed form does not exist in general. When $R_{2}=0$, it reduces to Aloha, whose throughput is maximized when $p=\frac{1}{N}$ in this case.

We could also choose $R_{1}=\frac{1}{2} \log \left(1+\frac{P}{(k-1) P+\sigma^{2}}\right)$ and $\omega_{1}=k$, where $k$ is an integer in $\{1, \ldots, N\}$. The average throughput is

$$
N R_{1} p \sum_{l=0}^{k-1}\left(\begin{array}{c}
N-1 \\
l
\end{array}\right) p^{l}(1-p)^{N-1-l}+N R_{2}(1-p)
$$

where $p$ is the probability of choosing $R_{1}$. When $k \ll N$ and $N \rightarrow+\infty$, (24) can be approximated as

$$
\xi e^{-\xi} R_{1} \sum_{l=0}^{k-1} \frac{1}{l !} \xi^{l}+N R_{2}
$$

where $\xi=N p$.

\section{RATE Splitting}

In this section, we extend our approach to rate splitting. We begin by considering the two user network as in Section II. Two virtual users, denoted as $U_{i}^{\prime}$ and $U_{i}^{\prime \prime}$ are created at node $i, i=1,2$, with power $\alpha P$ and $(1-\alpha) P$, respectively, where $\alpha$ is a parameter to be optimized. We take a suboptimal layering approach as in [8], where $U_{i}^{\prime \prime}$ 's packet is always decoded before $U_{i}^{\prime}$ 's packet. Thus, each virtual user as in Section II only needs to consider two rates, i.e., $U_{i}^{\prime}$ takes

$$
R_{k}^{\prime}(\alpha)=\frac{1}{2} \log \left(1+\frac{\alpha P}{(k-1) \alpha P+\sigma^{2}}\right), k=1,2,
$$

with probability $p_{k}^{\prime}$ and $U_{i}^{\prime \prime}$ takes

$$
R_{k}^{\prime \prime}(\alpha)=\frac{1}{2} \log \left(1+\frac{(1-\alpha) P}{(k-1)(1-\alpha) P+2 \alpha P+\sigma^{2}}\right), k=1,2,
$$

with probability $p_{k}^{\prime \prime}$. We choose $p_{1}^{\prime}=p^{\prime}, p_{2}^{\prime}=1-p^{\prime}$, and $p_{1}^{\prime \prime}=p^{\prime \prime}$, $p_{2}^{\prime \prime}=1-p^{\prime \prime}$. Note that our strategy is different from [8] where $U_{i}^{\prime}$ only transmits at rate $R_{2}^{\prime}(\alpha)$ and $U_{i}^{\prime \prime}$ only transmits at rate $R_{2}^{\prime \prime}(\alpha)$. The approach in [8] can be considered as a special case of our strategy by choosing $p^{\prime}=p^{\prime \prime}=0$.

As in Section II, the average throughput of multiple access MAC with rate splitting can be obtained as

$$
\begin{aligned}
& R_{\mathrm{drs}-\mathrm{mac}}\left(p^{\prime}, p^{\prime \prime}, \alpha\right) \\
= & 2\left(\left(1-p^{\prime \prime 2}\right)\left(\left(R_{1}^{\prime}(\alpha)+R_{2}^{\prime}(\alpha)\right) p^{\prime}\left(1-p^{\prime}\right)+R_{2}^{\prime}(\alpha)\left(1-p^{\prime}\right)^{2}\right)\right. \\
& \left.+\left(R_{1}^{\prime \prime}(\alpha)+R_{2}^{\prime \prime}(\alpha)\right) p^{\prime \prime}\left(1-p^{\prime \prime}\right)+R_{2}^{\prime \prime}(\alpha)\left(1-p^{\prime \prime}\right)^{2}\right) .
\end{aligned}
$$

Given $\alpha$, maximizing (28) over $p^{\prime}, p^{\prime \prime}$, we could obtain $R_{\mathrm{drs}-\mathrm{mac}}^{*}(\alpha)$. By performing a linear search over $\alpha$, we obtain the maximum total throughput $R_{\mathrm{drs}-\mathrm{mac}}^{*}$.

Fig. 1 compares the throughput of the proposed algorithm with that in [8] and Aloha with $\sigma^{2}=1$ and different $P$. We can see that the proposed schemes perform better than both Aloha and the one in [8]. The achievable rate of [8] is saturated when $P$ is large due to the lack of contention resolution mechanism. By using rate splitting, an additional performance gain is attained by using the proposed protocol compared with that without using rate splitting.

The proposed approach can be readily generalized to the case of choosing $M$ virtual users at each node forming $M$ layers. We still assume a layered decoding approach at the receiver by decoding the packets from virtual users at layer $m$ before decoding the packets from virtual users at layer $m-1$, where the users at layer $m$ are assigned power $\tilde{P}_{m}$ such that $\sum_{m=1}^{M} \tilde{P}_{m}=P$. Let $\phi_{m}$ be the probability that all layer $m$ users' packets are decoded correctly conditional on all the packets at layers less than $m$ are decoded successfully which can be computed by using the same idea as in Section III, and $R_{m}$ be the total throughput of users at layer $m$ given all lower layers' packets are decoded correctly. The total throughput of all virtual users can be written as $R_{\mathrm{drs}-\mathrm{mac}}=\sum_{m=1}^{M} R_{m} \Pi_{l=1}^{m-1} \phi_{l}$. The optimization of $\tilde{P}_{m}$ and the rate selection probabilities at each 


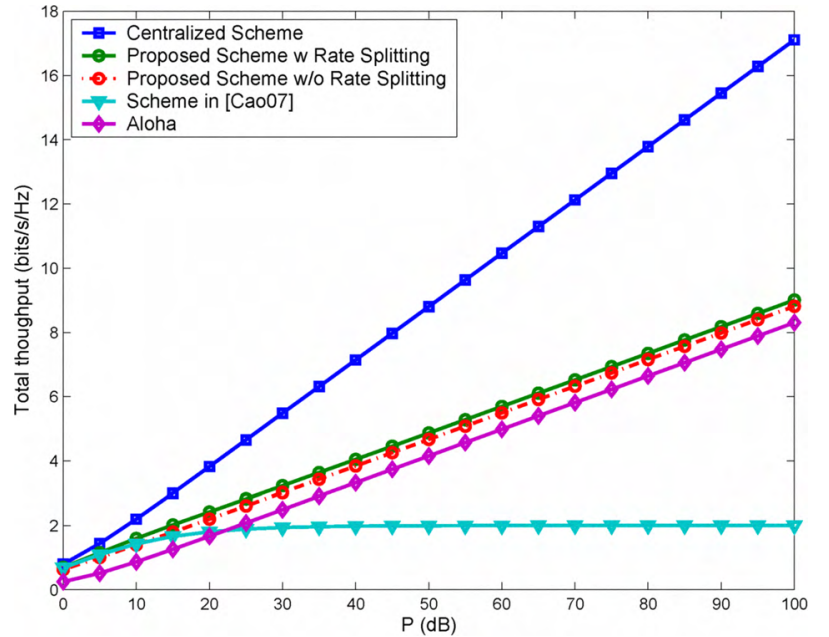

Fig. 1. Comparison of different schemes with $\sigma^{2}=1$ and different $P$ in a network with 2 users.

layer are coupled and are complicated to optimize. We thus take a suboptimal approach by decoupling the two optimizations. We first optimize over $\tilde{P}_{m}$ by ignoring each layer's impact on upper layers or assuming $\phi_{m}=1, m=1, \ldots, M$. We can show that $\tilde{P}_{m}=\frac{\sigma^{2}}{N}\left(1+\frac{N P}{\sigma^{2}}\right)^{\frac{M-m}{M}}\left(\left(1+\frac{N P}{\sigma^{2}}\right)^{\frac{1}{M}}-1\right), m=1, \ldots, M$, maximizes $\sum_{m=1}^{M} R_{m}$. After obtaining $\tilde{P}_{m}$, we optimize the rate assignment probability of layer $m$ backward from $m=M$ to $m=1$. When it comes to layer $m$, we need to maximize $\sum_{i=m}^{M} R_{i} \Pi_{l=m}^{i-1} \phi_{l}$, which can be solved similarly as in Section III.

\section{Simulation Results}

We now present simulation results on the proposed multiple access MAC protocol in Aloha type networks, which also apply to full duplex WLAN. We only consider the maximum achievable throughput without protocol overhead.

Fig. 2 compares the achievable throughput of different strategies as a function of $N$ when $P=10$ and $\sigma^{2}=1(\mathrm{SNR}=10 \mathrm{~dB})$. We compare the proposed protocol with centralized scheme and conventional Aloha.The "Equal Probability" throughput is obtained by $p_{k}=\frac{\alpha}{N-1}, k=1, \ldots, N-1$ and $p_{N}=1-\alpha$, where $\alpha=0.2011$ as in the proof of Theorem 1 . The optimized throughput of the proposed strategy is obtained by maximizing (12) via a local search around the "Equal Probability", which does not necessarily achieve the global maximum. In both equal probability and local search protocols, we set the number of transmission rates at each user to be $N$. The throughput of Aloha decreases as $N$ increases while that of the proposed protocol increases as $N$ increases. When $N=50$, the proposed protocol with local search achieves a 3.1951 times throughput gain over Aloha. Even with equal probability, the proposed protocol has a 2.2064 times throughput gain over Aloha at $N=50$. The ratio between the centralized scheme and the proposed strategy with local search decreases as $N$ increases. When $N=50$, the proposed strategy with local search attains 0.4580 throughput of the centralized scheme, which means that the lower bound (22) in Theorem 1 is very loose. We also include the achievable throughput of the proposed protocol with only $K$ transmission rates with $K$ being a finite number

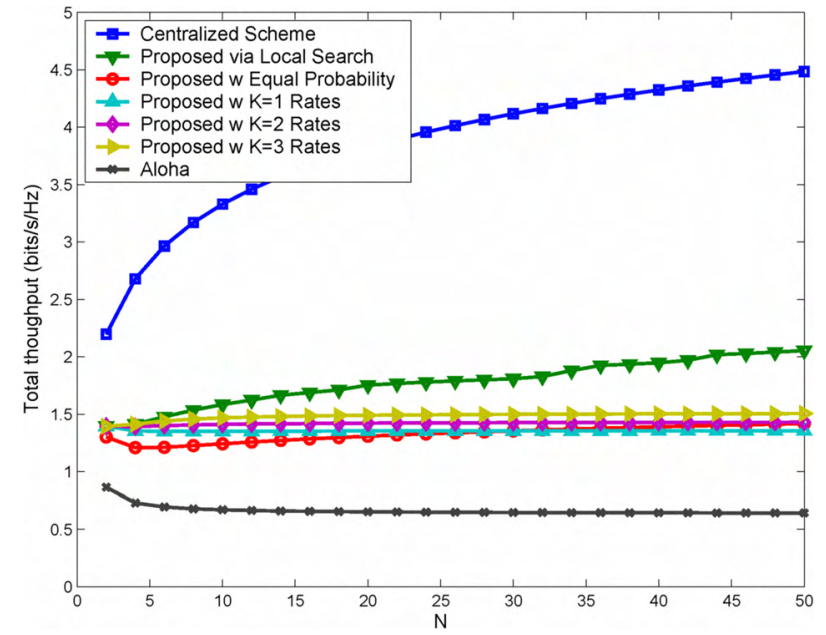

Fig. 2. Achievable throughput comparison of different strategies as a function of $N$ when $P=10$ and $\sigma^{2}=1(\mathrm{SNR}=10 \mathrm{~dB})$

as in Section III-C. The throughput of the proposed $K$-rate protocol increases with $K$. Even with $K=2$, the proposed protocol achieves a 3.4167 times throughput gain over Aloha at $N=50$. However, unlike the throughput obtained by using $N$ transmission rates which strictly increases as $N$ increases, the throughput obtained by using a finite number of transmission rates converges to a finite value as $N \rightarrow+\infty$ like Aloha.

\section{CONCLUSION}

In this paper, we have developed a new class of MAC protocol, which allows each user to transmit at different data rates within the multiple access capacity region. By using successive interference cancellation, multiple packets can be received simultaneously. In slotted Aloha type networks with Gaussian channels, we showed that the achievable sum rate of the new protocol is at least a constant fraction of the information theoretic limit. This approach was also extended to rate splitting.

\section{REFERENCES}

[1] N. Abramson, "The Aloha system-Another alternative for computer communications," in Proc. of Fall Joint Comput. Conf., Apr. 1970, pp. 281-285.

[2] L. Kleinrock and F. Tobagi, "Packet switching in radio channels: Part I-carrier sense multiple-access modes and their throughput-delay characteristics," IEEE Trans. Commun., vol. 23, no. 12, pp. 1400-1416, Dec. 1975.

[3] S. Ghez, S. Verdu, and S. C. Schwartz, "Stability properties of slotted Aloha with multipacket reception capability," IEEE Trans. Automat. Contr., vol. 33, no. 7, pp. 640-649, Jul. 1988.

[4] - "Optimal decentralized control in the random access multipacket channel," IEEE Trans. Automat. Contr., vol. 34, no. 11, pp. 1153-1163, Nov. 1989.

[5] T. Cover and J. Thomas, Elements of Information Theory, 1991.

[6] B. Rimoldi and R. Urbanke, "A rate-splitting approach to the Gaussian multiple-access channel," IEEE Trans. Inform. Theory, vol. 42, no. 2, pp. 364-375, Mar. 1996.

[7] M. Medard, J. Huang, A. Goldsmith, S. Meyn, and T. Coleman, "Capacity of time-slotted ALOHA packetized multiple-access systems over the AWGN channel," IEEE Trans. Wireless Commun., vol. 3, no. 2, pp. 486499, Mar. 2004.

[8] J. Cao and E. M. Yeh, "Asymptotically optimal multiple-access communication via distributed rate splitting," IEEE Trans. Inform. Theory, vol. 53, no. 1, pp. 304-319, Jan. 2007.

[9] T. Cui, L. Chen, and S. H. Low, "A game-theoretic framework for medium access control," IEEE J. Select. Areas Commun., vol. 26, no. 7, pp. 11161127, Sept. 2008. 